\title{
Improved dietary quality in women with previous gestational diabetes: secondary analysis of a randomised trial
}

\author{
S.L. O'Reilly ${ }^{1}$, V. Versace ${ }^{2}$, C. Yelverton ${ }^{1}$, E.D. Janus ${ }^{3}$ and J.A. Dunbar ${ }^{4}$ \\ ${ }^{1}$ School of Agriculture and Food Science, University College Dublin, Ireland, ${ }^{2}$ Deakin Rural Health, School of \\ Medicine, Deakin University, Geelong, Australia, ${ }^{3}$ School of Agriculture and Food Science, University College \\ Dublin, Ireland, ${ }^{4}$ General Internal Medicine Unit, Western Health and Department of Medicine, Melbourne \\ Medical School - Western Precinct, University of Melbourne, Australia and ${ }^{5}$ Centre for Population Health \\ Research, Deakin University, Australia
}

Women with previous gestational diabetes are seven times more likely to develop type 2 diabetes compared with those with normoglycaemic pregnancies ${ }^{(1)}$. Prevention of diabetes is possible; women with previous of gestational diabetes had $50 \%$ reduced incidence with a combination of moderate exercise and weight loss provided as a US diabetes prevention group-based program ${ }^{(2)}$. The dietary quality of women with gestational diabetes is reported to be low ${ }^{(3)}$ and we aimed to evaluate the effectiveness of a group-based lifestyle modification program improving dietary quality in women with previous gestational diabetes within their first postnatal year.

573 women were randomised to intervention $(n=284)$ or usual care $(n=289)$. The diabetes prevention intervention comprised one individual session, five group sessions, and two telephone sessions. Dietary data was collected at baseline and twelve months using a validated food frequency questionnaire, which was recoded using the Australian Recommended Food Score (ARFS) ${ }^{(3)}$. The ARFS category scores (vegetables, 22; fruit, grains, protein, 14 each; nuts/legumes, dairy, 7 each; fat, 1; alcohol, 2) are summed to provide a total ARFS of 74. Mixed model analyses investigated the effect of the intervention on ARFS. A per-protocol-set (PPS) was defined $a$ priori to exclude women who did not receive minimum exposure to the intervention.

The baseline total ARFS mean (SD) was 31.8 (8.9). No significant changes were seen in the total ARFS (intention-to-treat [ITT] and PPS analysis) over 12 months. The significant mean changes in ARFS sub-scores were as follows: $-0 \cdot 2$ nuts and legumes in intervention group $(95 \% \mathrm{CI}-0.36,-0.05)$ compared with +0.03 (SD) in usual care group $(95 \% \mathrm{CI}-0.13,0.18)$ (group by treatment interaction ITT, $p=0.04) ;+0.28$ dairy in intervention group $(95 \% \mathrm{CI} 0.08,0.48)$ compared with +0.02 in usual care group $(95 \% \mathrm{CI}$ $-0.14,0.18$ ) (group by treatment PPS interaction $p=0.05$ ); and +0.16 fats in intervention group (95\%CI 0.05 , 0.26$)$ compared with +0.02 in usual care group $(95 \% \mathrm{CI}-0.06,0.09)$ (group by treatment PPS interaction $p=0.03$ ).

Our results confirm that women with previous gestational diabetes have low diet quality. Intervention engagement influenced dietary quality within the PPS showing changes in dietary quality that aligns with group session attendance. Further research is needed to improve engagement with diabetes prevention programs, potentially through embedding interventions into standard care and increased use of mobile technology.

1. Bellamy L, Casas J-P, Hingorani AD, Williams D (2009) Type 2 diabetes mellitus after gestational diabetes: a systematic review and meta-analysis. Lancet 373(9677), 1773-9.

2. Ratner RE, Christophi CA, Metzger BE, Dabelea D, Bennett PH, Pi-Sunyer X et al. (2008) Prevention of Diabetes in Women with a History of Gestational Diabetes: Effects of Metformin and Lifestyle Interventions. J Clin Endocrinol Metab 93(12), 4774-9.

3. Morrison MK, Koh D, Lowe JM, Miller YD, Marshall AL, Colyvas K et al. (2012) Postpartum diet quality in Australian women following a gestational diabetes pregnancy. European journal of clinical nutrition 66(10), 1160-5. 